\title{
Stability Analysis of Switched Systems Using Lyapunov-Krasovskii Functionals
}

\author{
Sina Yamaç Çalışkan* Hitay Özbay** \\ Silviu-Iulian Niculescu*** \\ * Department of Electrical Engineering, UCLA, Los Angeles, CA \\ 90095-1594,USA; (e-mail: yamac@ucla.edu) \\ ** Department of Electrical and Electronics Engineering, Bilkent \\ University, 06800, Ankara, Turkey; (e-mail: hitay@bilkent.edu.tr) \\ *** Laboratoire des Signaux et Systèmes (UMR CNRS 8506) \\ CNRS-SUPELEC, 91192, Gif-sur-Yvette, France; (e-mail: \\ silviu.niculescu@lss.supelec.fr)
}

\begin{abstract}
Piecewise Lyapunov-Razumikhin functions are previously used for obtaining a lower bound for the dwell time of the switched time delay systems under the assumption that each candidate system is delay dependently stable. In this work, using Lyapunov-Krasovskii functionals, a less conservative lower bound for the dwell time is obtained. Improvement in the dwell time is illustrated with an example.
\end{abstract}

Keywords: Time Delay; Switched Systems; Lyapunov Methods

\section{INTRODUCTION}

Time delay systems appear in various engineering applications such as communication networks, chemical process control, transportation systems, Niculescu [2001]. Many analysis and control techniques are available for linear time delay systems where the parameters are fixed, see e.g. $\mathrm{Gu}$ et al. [2003] for detailed discussion and a review of the literature. When the system matrices and/or the delay(s) of a linear system change abruptly (such a jump may occur due to a sudden change in operating conditions or external effects), one must consider stability analysis techniques for switched systems. For delay-free switched systems see Bett and Lemon [1999], Colaneri et al. [2008], Hespanha et al. [2003], Hespanha [2004], Sun and Ge [2005], Skafidas et al. [1999], Yue and Han [2005] for available results and further references on this topic.

For a switched system let $t_{j}, j=1,2,3 \ldots$, denote the switching time instants and define $\Delta_{j}:=\left(t_{j+1}-t_{j}\right)$. Then, a lower bound (respectively, the average value) of the sequence $\Delta_{j}$ is called a dwell time (respectively, the average dwell time), for the switched system. If a system is switching arbitrarily between finitely many candidate systems, each of which are stable, then it is possible to guarantee stability of this switched system by putting a lower bound on the dwell time or on the average dwell time; see Morse [1996], Hespanha and Morse [1999] for related results on delay free switched systems. Extension of dwell time based stability results to time delay systems has been recently done in Kulkarni et al. [2004], Liu et al. [2008], Sun et al. [2003], Yan and Ozbay [2008], Yan et al. [2009], under various assumptions using different techniques. In particular, Yan and Ozbay [2008] has

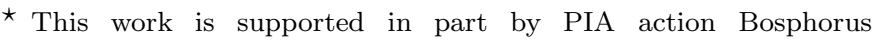
TUBITAK Grant No. 109E127 and EGIDE Project No. 22974WJ
}

obtained a dwell time by using a Lyapunov-Razhumikin technique for stability analysis of delay systems. In this work the results of Yan and Ozbay [2008] are improved by using a less conservative approach, namely the LyapunovKrasovskii method.

The paper is organized as follows. In Section 2 the problem definition and preliminary results are given. Main results are in Section 3. An illustrative example is given in Section 4 and concluding remarks are made in Section 5.

\section{PROBLEM DEFINITION AND PRELIMINARIES}

The general form of the retarded functional differential equation (RFDE) with time delay $\tau$ can be expressed as

$$
\dot{x}(t)=f\left(t, x_{t}\right)
$$

with appropriate initial condition $\phi(.) \in C\left([-\tau, 0], \mathbb{R}^{n}\right)$ and $f: \mathbb{R} \times C\left([-\tau, 0], \mathbb{R}^{n}\right) \rightarrow \mathbb{R}^{n}$ which maps bounded sets in $\mathbb{R} \times C\left([-\tau, 0], \mathbb{R}^{n}\right)$ to bounded sets in $\mathbb{R}^{n}$. In this equation, $x_{t}$ denotes the state defined by $x_{t}(\theta)=x(t+\theta)$ for $-\tau \leq \theta \leq 0$. Here, $C\left([a, b], \mathbb{R}^{n}\right)$ is the set of all continuous and bounded functions with domain $[a, b] \subset \mathbb{R}$ and range $\mathbb{R}^{n}$. Let $\|$.$\| be the Euclidean norm of a vector$ in $\mathbb{R}^{n}$ and for $f \in \mathbb{R} \times C\left([a, b], \mathbb{R}^{n}\right),|f|_{|t-\tau, t|}$ be the $\infty$ norm of $f$

$$
|f|_{|t-\tau, t|}:=\sup _{t-\tau \leq \theta \leq t}\|f(\theta)\|
$$

With the notations above, consider the following switched time delay systems:

$$
\Sigma_{t}=\left\{\begin{array}{l}
\dot{x}(t)=A_{q(t)} x(t)+\bar{A}_{q(t)} x\left(t-\tau_{q(t)}\right) \quad t \geq 0 \\
x_{0}(\theta)=\phi(\theta) \quad \forall \theta \in\left[-\tau_{\max }, 0\right],
\end{array}\right.
$$

where $x(t) \in \mathbb{R}^{n}$ is the state and $q(t)$ is the piecewise switching signal such that $q(t): \mathbb{R} \rightarrow \mathcal{F}$ where $\mathcal{F}:=$ $\{1,2, \ldots, l\}$. In other words, $q(t)=k_{j}, k_{j} \in \mathcal{F} \forall t \in$ $\left[t_{j}, t_{j+1}\right)$, where $j \in \mathbb{Z}^{+} \cup 0$ is the $j$ th switching time 
instant and $t_{j} \in \mathbb{R}_{+}$. From these definitions, it follows that the trajectory of $\Sigma_{t}$ in an arbitrary switching interval $\left[t_{j}, t_{j+1}\right)$ obeys

$$
\Sigma_{k_{j}}=\left\{\begin{array}{l}
\dot{x}(t)=A_{k_{j}} x(t)+\bar{A}_{k_{j}} x\left(t-\tau_{k_{j}}\right) \\
x_{t_{j}}(\theta)=\phi_{j}(\theta) \quad \forall \theta \in\left[-\tau_{k_{j}}, 0\right]
\end{array} \quad t \in\left[t_{j}, t_{j+1}\right)\right.
$$

where the initial condition $\phi_{j}(\theta)$ is defined as

$$
\phi_{j}(\theta)= \begin{cases}x\left(t_{j}+\theta\right) & -\tau_{k_{j}} \leq \theta<0 \\ \lim _{h \rightarrow 0^{-}} x\left(t_{j}+h\right) & \theta=0 .\end{cases}
$$

Let the triplet $\Sigma_{i}=\left(A_{i}, \bar{A}_{i}, \tau_{i}\right) \in \mathbb{R}^{n \times n} \times \mathbb{R}^{n \times n} \times \mathbb{R}^{+}$be the $i$ th candidate system of $(2)$ for some $i \in \mathcal{F}$. For every time instant $t, \Sigma_{t} \in \mathcal{A}=\left\{\Sigma_{i}: i \in \mathcal{F}\right\}$ where $\mathcal{A}$ is the set of all candidate systems. In the equation $(2), \tau_{\text {max }}=\max _{i \in \mathcal{F}} \tau_{i}$ is the maximal time delay of the candidate systems in $\mathcal{A}$.

The switched time delay system $\Sigma_{t}$ is stable Hespanha [2004] if there exists a strictly increasing continuous function $\bar{\alpha}: \mathbb{R}^{+} \rightarrow \mathbb{R}^{+}$with $\bar{\alpha}(0)=0$ such that

$$
\|x(t)\| \leq \bar{\alpha}\left(|x|_{\left[t_{0}-\tau_{\max }, t_{0}\right]}\right) \quad \forall t \geq t_{0} \geq 0
$$

along the trajectory of (2). The system is asymptotically stable if $\Sigma_{t}$ is stable and $\lim _{t \rightarrow \infty} x(t)=0$.

Lemma 1. (see Gu et al. [2003]). A given candidate system $\Sigma_{i}$ can be transformed into the following system denoted by $\Upsilon_{i}$

$$
\begin{aligned}
\dot{y}(t) & =\left(A_{i}+\bar{A}_{i}\right) y(t)-\int_{-2 \tau}^{\tau} \bar{A}_{i}^{2} y(t+\theta) d \theta \\
& -\int_{-\tau}^{0} \bar{A}_{i} A_{i} y(t+\theta) d \theta
\end{aligned}
$$

with the initial condition

$$
\psi_{i}(\theta)= \begin{cases}\phi(\theta) & -\tau_{i} \leq \theta<0 \\ \phi\left(-\tau_{i}\right) & -2 \tau_{i} \leq \theta<-\tau\end{cases}
$$

The construction of the model transformation is done by an appropriate integration on one delay interval, see e.g. Gu and Niculescu [2000]. Note that asymptotic stability of the system $\Upsilon_{i}$ implies asymptotic stability of the system $\Sigma_{i}$ but the reverse does not necessarily holds.

Lemma 2. (see $\mathrm{Gu}$ et al. [2003]). Suppose for a given triplet $\Sigma_{i} \in \mathcal{A}, \quad i \in \mathcal{F}$, there exists real symmetric matrices $P_{i}>0, S_{1 i}$ and $S_{2 i}$ that solves the LMI

$$
\left[\begin{array}{ccc}
M_{i} & -\tau P_{i} \bar{A}_{i} A_{i} & -\tau_{i} P_{i} \bar{A}_{i}^{2} \\
-\tau_{i} A_{i}^{T} \bar{A}_{i}^{T} P_{i} & -\tau_{i} S_{1 i} & 0 \\
-\tau_{i}\left(\bar{A}_{i}^{T}\right)^{2} P_{i} & 0 & -\tau_{i} S_{2 i}
\end{array}\right]<0
$$

where

$$
M_{i}=P_{i}\left(A_{i}+\bar{A}_{i}\right)+\left(A_{i}+\bar{A}_{i}\right)^{T} P_{i}+\tau_{i} S_{1 i}+\tau_{i} S_{2 i}
$$

then $\Upsilon_{i}$ is asymptotically stable. This guarantees the asymptotic stability of $\Sigma_{i}$ for all delays in the interval $\left[0, \tau_{i}\right]$.

Note that (8) implies $S_{1 i}>0, S_{2 i}>0$ and $A_{i}+\bar{A}_{i}$ is Hurwitz stable.

If all candidate systems of (2), $\Sigma_{i} \in \mathcal{A}$ are asymptotically stable satisfying (8), then the set $\mathcal{A}$ is denoted as $\tilde{\mathcal{A}}$. It is assumed that $\mathcal{A}=\tilde{\mathcal{A}}$ for the rest of the discussion. In this paper, sufficient condition that guarantees the asymptotic stability of the switched system (2) will be constructed using piecewise Lyapunov-Krasovskii functionals. One method in the stability analysis of switched systems is to find common Lyapunov function (CLF). In Zhai et al. [2003], CLFs are found for switched time delay systems assuming that each candidate system has the same time delay $\tau$, each candidate is assumed to be delay-independently stable, $A$ matrix is symmetric and $\bar{A}$ matrix is in the form $\delta I$. Even without these assumptions, method of finding CLFs are very conservative due to the fact that it is usually difficult to find a CLF for all the candidate systems, especially for time delay systems whose stability criteria are only sufficient in most cases. A recent work found asymptotic stability conditions using piecewise Lyapunov-Razumikhin functions Yan and Ozbay [2008]. In our work, by using piecewise Lyapunov-Krasovkii functionals, we will try to reduce the conservatism in Yan and Ozbay [2008].

\section{MAIN RESULTS}

For a given $\tau_{D}>0$, the switching signal set based on the dwell time $\tau_{D}$ is denoted as $S\left[\tau_{D}\right]$ where for any switching signal $q(t) \in S\left[\tau_{D}\right]$, the distance between any consecutive discontinuities of $q(t), t_{j+1}-t_{j}, j \in \mathbb{Z}^{+} \cup\{0\}$ is greater than $\tau_{D}$ Yan and Ozbay [2008], Hespanha and Morse [1999],Morse [1996]. Dwell time based switching is independent of the trajectory of the solutions Hespanha [2004]. Before presenting the main result of the paper, we need to recall some lemmas and prove some propositions which will be usefull in the proof of our main result.

Lemma 3. (see Hale and Verduyn Lunel [1993]). Suppose $u, v, w: \mathbb{R}^{+} \rightarrow \mathbb{R}^{+}$are continuous, nondecreasing functions, $u(0)=v(0)=0, w(s)>0$ for $s>0$. If there exists a continuous functional $V$ such that

$$
\begin{gathered}
u(\|x(t)\|) \leq V\left(t, x_{t}\right) \leq v\left(|x|_{[t-\tau, t]}\right) \quad \forall t \geq t_{0} \\
\dot{V}\left(t, x_{t}\right) \leq-w(\|x(t)\|) \quad \forall t \geq t_{0}
\end{gathered}
$$

then the solution $x=0$ of the RFDE (1) is uniformly asymptotically stable.

For functions defined in Lemma 3 , we say that $(V, u, v, w)$ is a stability quadruple for (1).

The arbitrary candidate system $\Sigma_{i}, i \in \mathcal{F}$ is a particular case of (1). Construct the following piecewise LyapunovKrasovskii functional for the transformed system $\Upsilon_{i}$ of the candidate system

$$
\begin{aligned}
V_{i}\left(t, x_{t}\right)= & x^{T}(t) P_{i} x(t)+\int_{-\tau_{i}}^{0} \int_{t+\theta}^{t} x^{T}(\xi) S_{1 i} x(\xi) d \xi d \theta \\
& +\int_{-2 \tau_{i}}^{-\tau_{i}} \int_{t+\theta}^{t} x^{T}(\xi) S_{2 i} x(\xi) d \xi d \theta
\end{aligned}
$$

where $P_{i}>0, S_{1 i}>0$ and $S_{2 i}>0$ are real symmetric matrices. This functional can be bounded by

$$
u_{i}(\|x(t)\|) \leq V_{i}\left(t, x_{t}\right) \leq v_{i}\left(|x|_{\left[t-2 \tau_{i}, t\right]}\right) \quad \forall t \geq t_{0}, \forall x \in \mathbb{R}^{n}
$$
where

and

$$
u_{i}(s)=\sigma_{\min }\left[P_{i}\right] s^{2}
$$

$$
v_{i}(s)=\left(\sigma_{\max }\left[P_{i}\right]+\frac{\tau_{i}^{2}}{2} \sigma_{\max }\left[S_{1 i}\right]+\frac{3 \tau_{i}^{2}}{2} \sigma_{\max }\left[S_{2 i}\right]\right) s^{2}
$$

Here $\sigma_{\min }[\cdot]$ and $\sigma_{\max }[\cdot]$ denote the minimum and maximum singular values, respectively. 
Proposition 4. For each time delay system $\Upsilon_{i}$ with Lyapunov Krasovskii functional (12), assume that (10) and (11) are satisfied for some $u$ and $v$ defined as in (13) and (14) respectively and a function $w_{i}: \mathbb{R}^{+} \rightarrow \mathbb{R}^{+}, w_{i}(s)>0$ for $s>0$, then we have the following result

$$
|x|_{\left[t_{m}-\tau_{i}, t_{m}\right]} \leq B_{i}|x|_{\left[t_{n}-2 \tau_{i}, t_{n}\right]} \forall t_{m} \geq t_{n}+\tau_{i}
$$

where

$$
B_{i}=\sqrt{\frac{\sigma_{\max }\left[P_{i}\right]+\frac{\tau_{i}^{2}}{2} \sigma_{\max }\left[S_{1 i}\right]+\frac{3 \tau_{i}^{2}}{2} \sigma_{\max }\left[S_{2 i}\right]}{\sigma_{\min }\left[P_{i}\right]}}
$$

Now consider the above stability quadruple $(V, u, v, w)$ with $\lim _{s \rightarrow \infty} u(s) \rightarrow \infty$. Then if $|\phi|_{\left[t_{0}-\tau, t_{0}\right]} \leq \delta_{1}$ and $\delta_{1}>0$, Lemma 3 implies that there exists $\delta_{2}>\delta_{1}>0$ such that $u\left(\delta_{2}\right)=v\left(\delta_{1}\right)$ and $\|x(t)\|<\delta_{2}$ for all $t>t_{0}$. For such a $\delta_{2}$, consider the following result.

Proposition 5. Suppose there exists a stability quadruple $(V, u, v, w)$ for $(1)$ with $\lim _{s \rightarrow \infty} u(s) \rightarrow \infty$. For an arbitrary $\eta, 0<\eta<\delta_{2},|\phi|_{\left[t_{0}-\tau, t_{0}\right]} \leq \delta_{1}<\delta_{2}$ implies

$$
\|x(t)\| \leq \eta \quad \forall t>t_{0}+T(\eta)
$$

where $T(\eta)=\frac{v\left(\delta_{1}\right)}{\gamma}, v$ is defined as in the Lemma 3 and $\gamma=\inf _{\eta \leq s \leq \delta_{2}} w(s)$.

Assume that for every transformed candidate system $\Upsilon_{i}$, each corresponding candidate system $\Sigma_{i}$ satisfies the Lemma 2 , in other words $\mathcal{A}=\tilde{\mathcal{A}}$. Let $\tau_{D}>\tau_{\max }$ and consider an arbitrary switching interval $\left[t_{j}, t_{j+1}\right)$ of the switching signal $q(t) \in S\left[\tau_{D}\right]$ where $q(t)=k_{j}, k_{j} \in \mathcal{F} \forall t \in$ $\left[t_{j}, t_{j+1}\right)$ and $t_{j} \in \mathbb{Z}^{+} \cup 0$ is the $j$ th switching time instant. The state variable $x_{j}(t)$ obeys (3) in this interval. Define $x_{j}\left(t_{j+1}\right)=\lim _{h \rightarrow 0^{-}} x\left(t_{j+1+h}\right)=x_{j+1}\left(t_{j+1}\right)$ based on the fact that $x(t)$ is continuous for $t \geq 0$. With this definition $x_{j}(t)$ is defined on the compact set $\left[t_{j}, t_{j+1}\right]$. The initial condition of $\Sigma_{k_{j}}$ is $\phi_{j}(t)=x(t)=x_{j-1}(t)$ where $t \in\left[t_{j}-\right.$ $\left.\tau_{k_{j}}, t_{j}\right]$ for $j \in \mathbb{Z}^{+}$. Initial condition of the transformed system $\Upsilon_{i}$ is $\phi_{i}(t)$ as defined before. Introduce now the Lyapunov-Krasovskii functional

$$
\begin{aligned}
V_{k_{j}}\left(t, x_{t}\right) & =x_{j}^{T}(t) P_{k_{j}} x_{j}(t) \\
& +\int_{-\tau_{k_{j}}}^{0} \int_{t+\theta}^{t} x_{j}^{T}(\xi) S_{1 k_{j}} x_{j}(\xi) d \xi d \theta \\
& +\int_{-2 \tau_{k_{j}}}^{-\tau_{k_{j}}} \int_{t+\theta}^{t} x_{j}^{T}(\xi) S_{2 k_{j}} x_{j}(\xi) d \xi d \theta .
\end{aligned}
$$

Then we have:

$$
\left\|x_{j}(t)\right\|^{2} \leq\left(\frac{\bar{\kappa}_{k_{j}}}{\kappa_{k_{j}}}+\frac{\tau_{k_{j}}^{2}}{2 \kappa_{k_{j}}} \bar{\chi}_{1 k_{j}}+\frac{3 \tau_{k_{j}}^{2}}{2 \kappa_{k_{j}}} \bar{\chi}_{2 k_{j}}\right)\left|x_{j}\right|_{\left[t-2 \tau_{k_{j}}, t\right]}
$$

for all $x_{j} \in \mathbb{R}^{n}, t \in\left[t_{j}, t_{j+1}\right]$ where $\kappa_{i}=\sigma_{\min }\left[P_{i}\right]$, $\bar{\kappa}_{i}=\sigma_{\max }\left[P_{i}\right], \bar{\chi}_{1 i}=\sigma_{\max }\left[S_{1 i}\right]$ and $\bar{\chi}_{2 i}=\sigma_{\max }\left[S_{2 i}\right]$.

Proposition 6. Let

$$
\begin{aligned}
W_{k_{j}}= & -\left(P_{k_{j}}\left(A_{k_{j}}+\bar{A}_{k_{j}}\right)+\left(A_{k_{j}}+\bar{A}_{k_{j}}\right)^{T} P_{k_{j}}\right) \\
& -\tau_{k_{j}}\left(R_{1 k_{j}}+R_{2 k_{j}}\right)
\end{aligned}
$$

where $R_{1 k_{j}}=R_{1 k_{j}}^{T}$ is the solution of the LMI

$$
\left[\begin{array}{cc}
S_{1 k_{j}}-R_{1 k_{j}} & -\tau_{k_{j}} P_{k_{j}} \bar{A}_{k_{j}} A_{k_{j}} \\
-\tau_{k_{j}} A_{k_{j}}^{T} \bar{A}_{k_{j}}^{T} P_{k_{j}} & -\tau_{k_{j}} S_{1 i}
\end{array}\right]<0
$$

and $R_{2 k_{j}}=R_{2 k_{j}}^{T}$ is the solution of the LMI

$$
\left[\begin{array}{cc}
S_{2 k_{j}}-R_{2 k_{j}} & -\tau_{k_{j}} P_{k_{j}} \bar{A}_{k_{j}}^{2} \\
-\tau_{k_{j}}\left(\bar{A}_{k_{j}}^{T}\right)^{2} P_{k_{j}} & -\tau_{k_{j}} S_{2 i}
\end{array}\right]<0
$$

then the upper bound on the derivative of the Lyapunov Krasovskii functional (18) can be set as

$$
\dot{V}_{k_{j}}\left(t, x_{t}\right) \leq-x_{j}^{T}(t) W_{k_{j}} x_{j}(t)
$$

Proof. Take the derivative of the Lyapunov Krasovskii functional with respect to time along the trajectory.

$$
\begin{aligned}
& \dot{V}_{k_{j}}\left(t, x_{t}\right)=x_{j}^{T}(t) D_{1 k_{j}} x_{j}^{T}(t)+ \\
& \int_{-\tau_{k_{j}}}^{0}\left[x_{j}^{T}(t) x_{j}^{T}(t+\theta)\right] D_{2 k j}\left[\begin{array}{c}
x_{j}(t) \\
x_{j}(t+\theta)
\end{array}\right] d \theta+ \\
& \int_{-2 \tau_{k_{j}}}^{-\tau_{k_{j}}}\left[x_{j}^{T}(t) x_{j}^{T}(t+\theta)\right] D_{3 k j}\left[\begin{array}{c}
x_{j}(t) \\
x_{j}(t+\theta)
\end{array}\right] d \theta
\end{aligned}
$$

where

$$
\begin{gathered}
D_{1 k_{j}}=P_{k_{j}}\left(A_{k_{j}}+\bar{A}_{k_{j}}\right)+\left(A_{k_{j}}+\bar{A}_{k_{j}}\right)^{T} P_{k_{j}}, \\
D_{2 k_{j}}=\left(\begin{array}{cc}
S_{1 k_{j}} & -\tau_{k_{j}} P_{k_{j}} \bar{A}_{k_{j}} A_{k_{j}} \\
-\tau_{k_{j}} A_{k_{j}}^{T} \bar{A}_{k_{j}}^{T} P_{k_{j}} & -\tau_{k_{j}} S_{1 i}
\end{array}\right), \\
D_{3 k_{j}}=\left(\begin{array}{cc}
S_{2 k_{j}} & -\tau_{k_{j}} P_{k_{j}} \bar{A}_{k_{j}}^{2} \\
-\tau_{k_{j}}\left(\bar{A}_{k_{j}}^{T}\right)^{2} P_{k_{j}} & -\tau_{k_{j}} S_{2 i}
\end{array}\right) .
\end{gathered}
$$

Add and subtract the term

$$
\int_{-\tau_{k_{j}}}^{0} x_{j}^{T}(t) R_{1 k_{j}} x_{j}(t) d \theta+\int_{-2 \tau_{k_{j}}}^{\tau_{k_{j}}} x_{j}^{T}(t) R_{2 k_{j}} x_{j}(t) d \theta
$$

to the right side of the equation (24) where $R_{1 k_{j}}$ and $R_{2 k_{j}}$ are the solutions of the LMIs (21) and (22) respectively. We obtain

$$
\begin{aligned}
& \dot{V}_{k_{j}}\left(t, x_{t}\right)=x_{j}^{T}(t) \tilde{D}_{1 k_{j}} x_{j}^{T}(t)+ \\
& \int_{-\tau_{k_{j}}}^{0}\left[x_{j}^{T}(t) x_{j}^{T}(t+\theta)\right] \tilde{D}_{2 k j}\left[\begin{array}{c}
x_{j}(t) \\
x_{j}(t+\theta)
\end{array}\right] d \theta+ \\
& \int_{-2 \tau_{k_{j}}}^{-\tau_{k_{j}}}\left[x_{j}^{T}(t) x_{j}^{T}(t+\theta)\right] \tilde{D}_{3 k j}\left[\begin{array}{c}
x_{j}(t) \\
x_{j}(t+\theta)
\end{array}\right] d \theta
\end{aligned}
$$

where

$$
\begin{gathered}
\tilde{D}_{1 k_{j}}=P_{k_{j}}\left(A_{k_{j}}+\bar{A}_{k_{j}}\right)+\left(A_{k_{j}}+\bar{A}_{k_{j}}\right)^{T} P_{k_{j}}+\tau_{k_{j}}\left(R_{1 k_{j}}+R_{2 k_{j}}\right), \\
\tilde{D}_{2 k_{j}}=\left(\begin{array}{cc}
S_{1 k_{j}}-R_{1 k_{j}} & -\tau_{k_{j}} P_{k_{j}} \bar{A}_{k_{j}} A_{k_{j}} \\
-\tau_{k_{j}} A_{k_{j}}^{T} \bar{A}_{k_{j}}^{T} P_{k_{j}} & -\tau_{k_{j}} S_{1 i}
\end{array}\right), \\
\tilde{D}_{3 k_{j}}=\left(\begin{array}{cc}
S_{2 k_{j}}-R_{2 k_{j}} & -\tau_{k_{j}} P_{k_{j}} \bar{A}_{k_{j}}^{2} \\
-\tau_{k_{j}}\left(\bar{A}_{k_{j}}^{T}\right)^{2} P_{k_{j}} & -\tau_{k_{j}} S_{2 i}
\end{array}\right) .
\end{gathered}
$$

Since $\tilde{D}_{2 k_{j}}$ and $\tilde{D}_{3 k_{j}}$ are negative definite,

$$
\begin{aligned}
& \dot{V}_{k_{j}}\left(t, x_{t}\right)=x_{j}^{T}(t) \tilde{D}_{1 k_{j}} x_{j}^{T}(t)+ \\
& \int_{-\tau_{k_{j}}}^{0}\left[x_{j}^{T}(t) x_{j}^{T}(t+\theta)\right] \tilde{D}_{2 k j}\left[\begin{array}{c}
x_{j}(t) \\
x_{j}(t+\theta)
\end{array}\right] d \theta+ \\
& \int_{-2 \tau_{k_{j}}}^{-\tau_{k_{j}}}\left[x_{j}^{T}(t) x_{j}^{T}(t+\theta)\right] \tilde{D}_{3 k j}\left[\begin{array}{c}
x_{j}(t) \\
x_{j}(t+\theta)
\end{array}\right] d \theta \\
& \leq x_{j}^{T}(t) \tilde{D}_{1 k_{j}} x_{j}^{T}(t)=-x_{j}^{T}(t) W_{k_{j}} x_{j}(t)
\end{aligned}
$$


Best choice of $W_{k_{j}}$ is obtained from the following optimization problem. Maximize $r$ over all $r \in \mathbb{R}^{+}$and symmetric matrices $P_{k_{j}}, R_{1 k_{j}}, R_{2 k_{j}}, S_{1 k_{j}}, S_{2 k_{j}}$ subject to LMIs (21), (22) and additional constraints

$$
\left[\begin{array}{ccc}
M_{i} & -\tau P_{k_{j}} \bar{A}_{k_{j}} A_{k_{j}} & -\tau_{k_{j}} P_{k_{j}} \bar{A}_{k_{j}}^{2} \\
-\tau_{k_{j}} A_{k_{j}}^{T}{\overline{k_{k}}}^{T} P_{k_{j}} & -\tau_{i} S_{1 k_{j}} & 0 \\
-\tau_{k_{j}}\left(\bar{A}_{k_{j}}^{T}\right)^{2} P_{k_{j}} & 0 & -\tau_{i} S_{2 k_{j}}
\end{array}\right]<0,
$$

$P_{k_{j}}\left(A_{k_{j}}+\bar{A}_{k_{j}}\right)+\left(A_{k_{j}}+\bar{A}_{k_{j}}\right)^{T} P_{k_{j}}+\tau_{k_{j}}\left(R_{1 k_{j}}+R_{2 k_{j}}\right)+r I \leq 0$. The matrices $P_{k_{j}}, R_{1 k_{j}}, R_{2 k_{j}}, S_{1 k_{j}}$ and $S_{2 k_{j}}$ are obtained from the solution of this optimization problem. From these matrices we can determine $\sigma_{\min }\left[P_{i}\right], \sigma_{\max }\left[P_{i}\right], \sigma_{\max }\left[S_{1 i}\right]$, $\sigma_{\max }\left[S_{2 i}\right]$ and

$W_{k_{j}}^{*}=P_{k_{j}}\left(A_{k_{j}}+\bar{A}_{k_{j}}\right)+\left(A_{k_{j}}+\bar{A}_{k_{j}}\right)^{T} P_{k_{j}}+\tau_{k_{j}}\left(R_{1 k_{j}}+R_{2 k_{j}}\right)$

Select $w(s)$ in Lemma 3 as $w(s)=\varpi_{k_{j}} s^{2}$ where $\varpi_{k_{j}}=$ $\sigma_{\min }\left[W_{k_{j}}^{*}\right]>0$. With this selection, (11) is satisfied.

Assume $\left|\phi_{j}(t)\right|_{\left[t_{j}-\tau_{j}, t_{j}\right]} \leq \delta_{j}$. For an arbitrary $\alpha$ with $0<\alpha<1$, let $\eta=\alpha \delta_{j}$ in Proposition 5. With this selection of $\eta$ and $\delta_{j}=\delta_{1}$, we have $0<\eta=\alpha \delta_{j}<\delta_{1}<\delta_{2}$. Using the Proposition 5, we have

where

$$
\left\|x_{j}(t)\right\| \leq \alpha \delta_{j} \quad \forall t \geq t_{j}+T_{j}
$$

$$
T_{j}=\frac{v\left(\delta_{j}\right)}{\gamma}=\frac{\left(\bar{\kappa}_{j}+\frac{\tau_{j}^{2}}{2} \bar{\chi}_{1 j}+\frac{3 \tau_{j}^{2}}{2} \bar{\chi}_{2 j}\right)}{\alpha^{2} \varpi_{j}}
$$

Equation (27) implies

Let

$$
|x|_{\left[t_{j}+T_{j}, t_{j+1}\right]} \leq \alpha \delta_{j}
$$

$$
\begin{gathered}
\lambda=\max _{i \in \mathcal{F}} \frac{\sigma_{\max }\left[P_{i}\right]+\frac{\tau_{i}^{2}}{2} \sigma_{\max }\left[S_{1 i}\right]+\frac{3 \tau_{i}^{2}}{2} \sigma_{\max }\left[S_{2 i}\right]}{\sigma_{\min }\left[P_{i}\right]} \\
\mu=\max _{i \in \mathcal{F}} \frac{\bar{\kappa}_{i}}{\varpi_{i}} \\
\rho_{1}=\max _{i \in \mathcal{F}} \frac{\tau_{\max }^{2} \bar{\chi}_{1 i}}{2 \varpi_{i}}
\end{gathered}
$$

and

$$
\rho_{2}=\max _{i \in \mathcal{F}} \frac{3 \tau_{\max }^{2} \bar{\chi}_{2 i}}{2 \varpi_{i}}
$$

Define

Note that

$$
T^{*}=\frac{\mu+\rho_{1}+\rho_{2}}{\alpha^{2}}
$$

$T^{*}>T_{j}=\frac{v\left(\delta_{j}\right)}{\gamma}=\frac{\left(\bar{\kappa}_{j}+\frac{\tau_{j}^{2}}{2} \bar{\chi}_{1 j}+\frac{3 \tau_{j}^{2}}{2} \bar{\chi}_{2 j}\right)}{\alpha^{2} \varpi_{j}} j=0,1,2, \ldots$

Let the dwell time to be $\tau_{D}=T^{*}+2 \tau_{\max }$. Recall that $t_{j+1}-t_{j}>\tau_{D}$. Thus $t_{j+1}-t_{j}>T^{*}+2 \tau_{\max }>T^{*}+$ $2 \tau_{j+1}>T_{j}+2 \tau_{j+1}$. Also note that $\left|\psi_{j+1}(t)\right|=\left|x_{j}(t)\right|$ where $t \in\left[t_{j+1}-2 \tau_{j+1}, t_{j+1}\right]$. Thus we have

$$
\begin{aligned}
\left|\psi_{j+1}\right|_{\left[t_{j+1}-2 \tau_{j+1}, t_{j+1}\right]} & =\left|x_{j}\right|_{\left[t_{j+1}-2 \tau_{j+1}, t_{j+1}\right]} \\
& \leq\left|x_{j}\right|_{\left[t_{j}+T_{j}, t_{j+1}\right]} \leq \alpha \delta_{j}:=\delta_{j+1}
\end{aligned}
$$

and $\delta_{0}$ is defined as $\delta_{0}:=|\psi|_{\left[-2 \tau_{\max }, 0\right]}=|\phi|_{\left[-\tau_{\max }, 0\right]} \geq$ $|\phi|_{\left[-\tau_{k_{0}}, 0\right]}$. Therefore we obtain a convergent sequence $\delta_{i}$ where $\delta_{i}=\alpha^{i} \delta_{0}$ with $i=0,1,2, \ldots$.

Proposition 4 implies

$$
\begin{aligned}
& |x|_{\left[t, t+\tau_{i}\right]} \leq \\
& \sqrt{\frac{\sigma_{\max }\left[P_{i}\right]+\frac{\tau_{i}^{2}}{2} \sigma_{\max }\left[S_{1 i}\right] \frac{3 \tau_{i}^{2}}{2} \sigma_{\max }\left[S_{2 i}\right]}{\sigma_{\min }\left[P_{i}\right]}}|x|_{\left[t_{n}-2 \tau_{i}, t_{n}\right]}
\end{aligned}
$$

$\forall t \geq t_{j}$. Thus

$$
\begin{aligned}
\sup _{t \in\left[t_{j}, t_{j+1}\right]}\left\|x_{j}(t)\right\| & \leq \sup _{t \in\left[t_{j}, t_{j+1}\right]}\left|x_{j}(t)\right|_{\left[t, t+\tau_{k_{j}}\right]} \\
& \leq \sqrt{\lambda}\left|x_{j}\right|\left[t_{j}-2 \tau_{k_{j}}, t_{j}\right] \\
& \leq \sqrt{\lambda} \delta_{j}=\alpha^{j} \sqrt{\lambda} \delta_{0}
\end{aligned}
$$

which implies the asymptotic stability of the transformed switched time delay system $\Upsilon_{t}$ with the switching signal $q(t) \in S\left[\tau_{D}\right]$. Asymptotic stability of the transformed switched time delay system implies the asymptotic stability of the switched time delay system $\Sigma_{i}$. Thus we can state our final result as follows.

Theorem \%. Under the assumptions stated above, let the dwell time be defined by $\tau_{D}=T^{*}+2 \tau_{\max }$ where

$$
T^{*}=\frac{\mu+\rho_{1}+\rho_{2}}{\alpha^{2}},
$$

then the system $\Sigma_{t}$ is asymptotically stable for any switching rule $q(t) \in S\left[\tau_{D}\right]$.

\section{EXAMPLE}

The system given below is taken from Yan and Ozbay [2008] for comparison purposes. Let $\Sigma_{1}$ be

$$
A_{1}=\left[\begin{array}{cc}
-2 & 0 \\
0 & -0.9
\end{array}\right], \quad \bar{A}_{1}=\left[\begin{array}{cc}
-1 & 0 \\
-0.5 & -1
\end{array}\right], \quad \tau_{1}=0.3 .
$$

Let $\Sigma_{2}$ be

$$
A_{2}=\left[\begin{array}{cc}
-1 & 0.5 \\
0 & -1
\end{array}\right], \quad \bar{A}_{2}=\left[\begin{array}{cc}
-1 & 0 \\
0.1 & -1
\end{array}\right], \quad \tau_{2}=0.6
$$

Initial conditions for this switched system are selected as

$$
\phi(t)=\left[\begin{array}{l}
5 \cos \left(\frac{\pi}{2.4} t+\frac{\pi}{6}\right) \\
5 \sin \left(\frac{\pi}{2.4} t+\frac{\pi}{6}\right)
\end{array}\right] \quad \forall t \in[-0.6,0] .
$$

In the paper Yan and Ozbay [2008], dwell time for this system is found to be $\tau_{D}=6.52 \mathrm{sec}$. Using Theorem 7 , a dwell time is found as $\tau_{D}=1.2+\frac{2.15}{\alpha^{2}}$ seconds for a fixed $\alpha$. Note that system is stable for all $\alpha \in(0,1)$. For $\alpha>0.48$ our dwell time result is smaller than $6.52 \mathrm{sec}$. Let us take $\alpha=0.99$. This implies $\tau_{D}=3$.4. In Figure 1 , it can be observed that for a switching signal with $\tau_{D}=3.4$, the system is still stable. Thus result of Yan and Ozbay [2008] can be improved. The state trajectories for $\tau_{D}=3.4$ case are given in Figure 1.

\section{CONCLUSIONS}

In this work, using a piecewise Lyapunov-Krasovskii functional, a dwell time is obtained for the asymptotic stability of time-delayed switched systems. The new dwell time expression is less conservative than the one found in Yan and Ozbay [2008], where Lyapunov-Razumikhin method was used. We should emphasize that dwell time expression derived here comes from solutions of certain LMIs. It is possible to improve this result by different choices of 

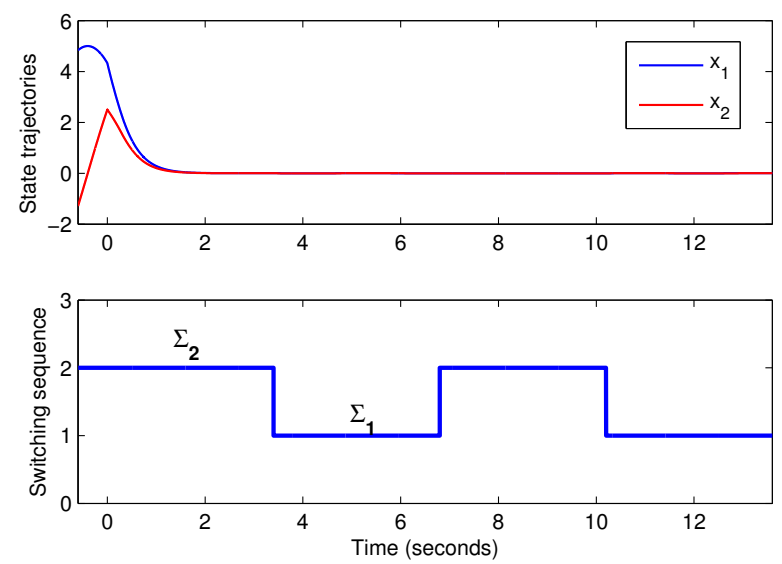

Fig. 1. State trajectories of the switched system.

Lyapunov-Krasovskii functionals. However, what is the best functional for dwell time minimization is a difficult question; we leave this as an open problem for future studies.

\section{REFERENCES}

C. J. Bett, and M. D. Lemmon. Bounded amplitude performance of switched LPV systems with applications to hybrid systems, Automatica, 35, pp. 491-503, 1999.

P. Colaneri, J. C. Geromel, and A. Astolfi. Stabilization of continuous-time switched nonlinear systems, Systems and Control Letters, 57, pp. 95-103, 2008.

K. Gu, V. L. Kharitanov, and J. Chen. Stability and robust stability of time delay systems, Birkhauser, Boston, 2003.

K. Gu and S.-I. Niculescu. Further Remarks on Additional Dynamics in Various Model Transformations of Linear Delay Systems. Proceedings of the 2000 American Control Conference Chicago, IL, pp. 4368-4372, June 2000.

J. Hale and S. Verduyn Lunel. Introduction to Functional Differential Equations, Springer-Verlag, New York, 1993.

J. Hespanha, and S. Morse. Stability of switched systems with average dwell time. Proceedings of the 38th IEEE Conference on Decision and Control, Phoenix, AZ, pp. 2655-2660, 1999.

J. Hespanha, D. Liberzon, and S. Morse. Overcoming the limitations of adaptive control by means of logic-based switching, Systems Control Lett., 49, pp. 49-65, 2003.

J. Hespanha. Uniform stability of switched linear systems: Extension of LaSalle's invariance principle. IEEE Trans. Automatic Control, 49, pp. 470-482, 2004.

V. Kulkarni, M. Jun, and J. Hespanha. Piecewise quadratic Lyapunov function for piecewise affine timedelay systems, Proceedings of the 2004 American Control Conference, Boston, MA, USA, pp. 3885-3889, 2004.

J. Liu, X. Liu, W.-C. Xie. Delay dependent robust control for uncertain switched systems with time delay, Nonlinear Analysis: Hybrid Systems, 2, pp. 81-95, 2008.

A. S. Morse. Supervisory control of families of linear setpoint controlers, part 1: Exact matching, IEEE Trans. Automatic Control, 41, pp. 1413-1431, 1996.
S.-I. Niculescu, Delay Effects on Stability: A Robust Control Approach, Lecture Notes in Control and Inform. Sci. 269, Springer-Verlag, Heidelberg, 2001.

E. Skafidas, R. J. Evans, A. V. Savkin, and I. R. Petersen. Stability results for switched control systems, Automatica, 35, pp. 553-564, 1999.

Z. Sun, and S. S. Ge. Analysis and synthesis of switched linear control systems, Automatica, 41, pp. 181-195, 2005.

Y. Sun, A Michel, and G. Zhai. Stability of discontinuous retarded functional differential equations with applications to delay systems, Proceedings of the American Control Conference, Denver, CO, pp. 3387-3392, 2003.

P. Yan, and H. Özbay. Stability analysis of switched time delay systems. SIAM Journal of Control Optim., Vol. 47, pp. 936-949, 2008.

P. Yan, H. Özbay, and M. Şansal. A switching controller approach to stabilization of parameter varying time delay systems, Joint 48th IEEE Conference on Decision and Control and 28th Chinese Control Conference, Shanghai, P. R. China, pp. 7222-7226, 2009.

D. Yue, and Q.-L. Han. Delay-dependent exponential stability of stochastic systems with time-varying delay, nonlinearity, and Markovian switching, IEEE Trans. Automatic Control, 50, pp. 217-222, 2005.

G. Zhai, Y. Sun, X. Chen and A. Michel. Stability and $L^{2}$ gain analysis for switched symmetric systems with time delay. Proceedings of the American Control Conference, Denver, CO, pp. 2682-2687, 2003. 